\title{
The Choice of Surgical Tactics for the Treatment of Children with Destructive Pathological Dislocation of the Hip after Hematogenous Osteomyelitis
}

\author{
Akhror Makhmutovich Djuraev \\ Ministry of Health of the Republic of Uzbekistan, Uzbekistan \\ Khojaakhmed Shaykhislamovich Alpisbaev \\ Ministry of Health of the Republic of Uzbekistan, Uzbekistan \\ Corresponding author email:xojaahmed@mail.ru \\ Elyar Abduvalievich Tapilov \\ Ministry of Health of the Republic of Uzbekistan, Uzbekistan
}

\begin{abstract}
A surgical approach to the treatment of destructive pathological dislocation of the hip in children has been substantiated, depending on age, the severity of destruction of the proximal femur and acetabulum. Reconstructive - restorative operations in most patients ensure the stability of the hip joint and thereby improve the patient's gait and statics, reduce the pelvic misalignment and eliminate the Trendelenburg symptom. The experience of surgical treatment of patients with pathological hip dislocations has shown that the most favorable outcomes are achieved with the use of open reduction of the stump of the head or neck of the femur with intertrochanteric detorsion shortening and varizing osteotomy with rotational pelvic osteotomy according to Salter and acetabular plasty according to Pemberton.
\end{abstract}

Keywords---articulo-trochanteric distance, hip joint, osteomyelitis, pathological hip dislocation

\section{Introduction}

Orthopedic complications after acute hematogenous osteomyelitis are caused by different severity variants of damage to the cartilaginous and bone structures of the components of the hip joint. The formation of orthopedic complications is based on: impaired cartilage ossification, impaired growth of the femoral head and acetabulum, destruction of the cartilaginous and bone tissue of the acetabulum and the proximal femur. Pathological dislocation of the hip is one of the most severe complications of acute hematogenous osteomyelitis. As a result of the spread of the inflammatory process to the pineal gland, the adjacent joint is involved in the process in 50-96\% of patients, which leads to a violation of its function and the formation of pathological dislocation. The epimetaphyseal (54.5\%) and metaphyseal (39.6\%) zones of long bones are most often affected (Belokrylov et al., 2007), and the femur (38$46 \%$ ) is most often affected by localization. Dysfunction or destruction of the epiphyseal cartilaginous plate leads to a shortening of the limb that progresses during the child's growth. The pathology is complicated by cicatricial degeneration of soft tissues and static deformities. In children, the severity of the disease in the long term is aggravated by the defeat of the meta-epiphyseal zones, which leads to a violation of the further formation of the musculoskeletal system. They include premature closure or destruction of growth zones, destruction of bone and cartilaginous structures of the hip joint, violation of articular relationships. Surgical treatment of the consequences of hematogenous osteomyelitis is an extremely complex and debatable problem of pediatric orthopedics (Baghdadi et al., 2012). A large number of reconstructive intervention techniques are known, aimed at the formation of the hip joint. For the formation of the supporting surface of the thigh, depending on the degree of destruction, the stump of the neck, the medial part or apex of the greater trochanter, and the lesser trochanter are used (Belokrylov et al., 
2007). In the surgical treatment of PVB, the primary task is to restore the support function of the limb while maintaining mobility in the joint, followed by solving the problem of eliminating secondary deformities and equalizing the length of the lower limbs (Belokrylov et al., 2007). Depending on the degree of destructive changes, therapeutic measures, as a rule, include correction of the deformity of the proximal femur, compensation for the lost elements of the femur, increased supportability, restoration, and stabilization of articular relationships (Forlin \& Milani, 2008).

Until now, the age of the patient in which he should be operated on, the choice of a surgical technique aimed at stabilizing the hip joint, and the choice of a segment of the lower limb to compensate for its shortening are subject to discussion. One of the controversial issues is the definition of age limits for the use of these interventions (Goel \& Logani, 2003). In children who have undergone septic coxitis in the first three months, reconstructive operations worsen the patient's condition, as they lead to the formation of stiff painful joints (Chang \& Huang, 1997). In this regard, the authors recommend refraining from any surgical interventions, with the exception of "epiphysiodesis" and limb lengthening (Baghdadi et al., 2012). Some authors note good and satisfactory positive outcomes of reconstructive operations performed at the age of 8-13 years (Sokolovsky \& Sokolovsky, 1997).Yu.E. Garkavenko report about 73\% positive long-term results of hip arthroplasty performed in school and adolescent patients when the disorders are already pronounced (Garkavenko, 2017). However, most authors agree that a favorable outcome can be achieved only when the operation is performed at an early age (Baghdadi et al., 2012). Choi et al. admit the implementation of reconstructive interventions only in children under six years of age (Choi et al., 2005). In older patients, due to the risk of developing a stiff painful joint, they prefer supporting osteotomy of the hip (Kruczynski, 1996, Avascular necrosis of the proximal femur in developmental dislocation of the hip: Incidence, risk factors, sequelae and MR imaging for diagnosis and prognosis. Acta Orthop Scand). This once again confirms the need for a differentiated approach to the treatment of children with this disease and the choice of the optimal tactics of surgical treatment. The aim of the study was to determine the tactics of surgical treatment of deformity of the proximal femur in pathological dislocation of the hip.

\section{Materials and Research Methods}

The work is based on the results of observation and treatment of 127 patients with pathological dislocation of the hip treated at the Department of Hip Joint Pathology of the Republican Center for Pediatric Orthopedics of the Ministry of Health of the Republic of Uzbekistan from 2010 to 2020. Among these patients, there were 70 boys and 57 girls, aged 4 to 14 years. The work used: clinical, radiological, magnetic resonance tomographic, and multispiral computed tomographic research methods (Abrishami et al., 2010; Choi et al., 2006; Lew \& Waldvogel, 2004). Typical indicators of the hip joint, characterizing the shape and size of the head, the condition of the neck, the shape and depth of the depression, the nature of the articular relationships, acetabular coefficient, indicators of the proximal femur, cervico-diaphyseal angle, anteversion angle, Alsberg angle, degree of bone coverage and Shenton's line were evaluated. For a detailed analysis of the ongoing anatomical changes, additional indicators were assessed characterizing the ratio of the head and greater trochanter of the femur in the frontal plane:

a) articulo-trochanteric distance (ATD) - the distance from the apex of the greater trochanter to the upper pole of the femoral head $(\mathrm{mm})$. Normally $9.5-26.6 \mathrm{~mm}$.

b) trochanter-to-trochanter distance (TTD) - the distance from the apex of the greater trochanter to the middle of the lesser trochanter along a line parallel to the anatomical axis of the femur. The indicator reflects the growth of the greater trochanter and does not depend on the growth of the pineal gland. Normally 28.8-72.6 mm.

c) the distance from the lesser trochanter to the upper pole of the femoral head (lessertrochanter-to-articular surface distance, LTA). The indicator reflects the growth of the epiphysis and femoral neck and does not depend on the growth of the greater trochanter (Figure 2).

\section{The main findings and results}

The data obtained were used to plan the surgical intervention. The optimal age for surgical treatment of pathological dislocation of the hip, according to our data, is 4-5 years of age of the child, since by this time the process of ossification of the structures of the hip joint ends in most patients when secondary deformities have not yet formed and the regeneration processes are well expressed (Dobbs et al., 2003; Krys'-Pugach, 2008; Menschik, 1997). The planning of surgical intervention in patients was based on the assessment of violations of the anatomical relationships in the hip joint, its function, deformation of the proximal end of the femur, and the state of the glenoid 
cavity, which allows the formation of the required cavity size, as well as violations of the size, shape and integrity of bones and was carried out taking into account the identified symptom complexes and the age of the patient. Contraindications to surgical treatment: the danger of latent infection in patients who underwent infectious coxitis 1.5 - 2 years ago and do not have repeated manifestations of the inflammatory process. All surgical interventions were accompanied by a revision of the hip joint. We did not use extra-articular surgical interventions. The choice of the method of surgical intervention depended on the patient's age, the extent of the destruction of the head, the femoral neck of the acetabulum, and the degree of displacement of the hip (Rozbruch et al., 2005; Philippon et al., 2009; Pidhaietskyi et al., 2021). In case of destructive hip dislocation, differentiated surgical tactics were used depending on the severity of destruction of the proximal femur and acetabulum. We carried out the following complex reconstructive - restorative operations:

- open reduction of the stump of the head or neck of the femur with intertrochanteric detorsion-shortening and varizing osteotomy in 64 children;

- open reduction of the stump of the head or neck of the femur with intertrochanteric detorsion-shortening and varizing osteotomy with acetabular plasty according to Pemberton;

- rotational osteotomy of the pelvis according to Salter in 34 children;

- open reduction of the stump of the head and neck with lengthening of the latter and transposition of the hypertrophied greater trochanter with gluteal muscles in the caudal direction with fixation with screws in 29 children.

The purpose of such operations is to improve the supporting and dynamic function of the affected limb, to eliminate the vicious position of the limb and the most severe of the symptoms of dislocation - the Trendelenburg symptom, to reduce the shortening of the limb and, if possible, increase the range of motion in the joint. Example \# 1. Patient M.A., no. I / b 1645/411, was admitted to the Department of Hip Pathology on May 21, 2018, with complaints of lameness in the left leg, minor pain when walking, shortening of the left lower limb, and limitation of movement in the left hip joint. Objectively, lameness when walking in the left leg and limitation of movement in the left hip joint was observed. Movement in the hip joints: flexion - 1200/900; abduction - 600/200; internal rotation-400/100; external rotation - 450/150. Trendelenburg's symptom is positive on the left. The greater trochanter of the left femur protrudes and is located above the Roser-Nelaton line. The length of the lower extremities: the left lower limb was shortened by $2.0 \mathrm{~cm}$. The plain radiograph showed a pathological destructive subluxation of the left femur with deformity of the proximal femur: "coxabreva" and the relatively high standing of the greater trochanter. The Shenton and Calvet lines are interrupted on the left. D-z.: Pathological destructive subluxation of the left femur with deformation of the proximal femur. Coxabreva and the relative high standing of the greater trochanter. Figure 1 (a, b, c).

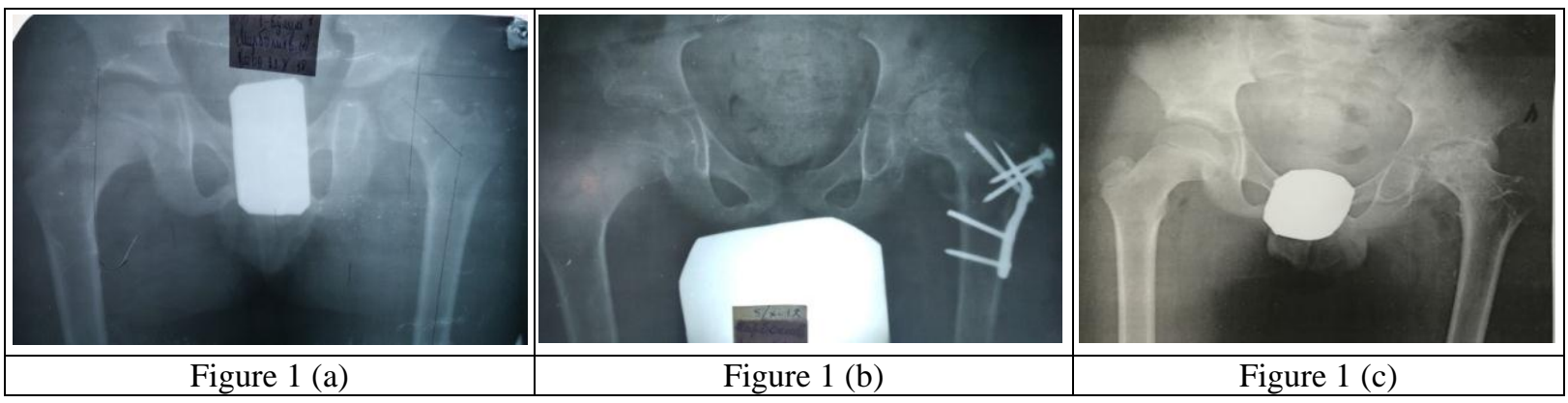

Figure 1 (a): Pathological subluxation of the left femur with deformity of the proximal femur. Coxabreva and the relative high standing of the greater trochanter. The Shenton and Calvet lines are interrupted on the left.

Figure 1 (b); SPO. Open centering of the left femur head with intertrochanteric detorsion-valgus and "lengthening" neck osteotomy and transposition of the greater trochanter of the femur according to the above technique.

Figure 1 (c); Result in 1 year 8 months after surgical treatment.

On May 24, 2018, an open centering of the left femur head was performed with intertrochanteric detorsion-valgus and "lengthening" osteotomy of the neck and transposition of the greater trochanter of the femur according to the above-described technique. After the operation, a hip plaster cast was applied. 1.5 months after the operation, the hip 
plaster cast was removed and a plaster cast "boot with a deratator" was applied to the right lower limb for 2-2.5 months. The patient received courses of rehabilitation measures. with the inclusion of exercise therapy of the hip joints. On October 16, 2018, under combined anesthesia, the metal structures were removed from the proximal end of the left femur. 3-4 months after the removal of the metal structures, the patient was allowed to walk with crutches.

Figure 1 (c). Plain X-ray: the head of the left femur is in the cavity, the coverage is good, the neck of the femur is lengthened, the greater trochanters of the femur are in line. From the present time the patient walks independently, chromate disappeared, movements in the hip joints: flexion - 1200/1000; abduction - 600/500; internal rotation400/200; external rotation - 450/350. Trendelenburg's symptom is negative. The greater trochanter of the left femur does not protrude and is located on the Roser-Nelaton line. The length of the lower limbs is the same. Clinical example No. 2. Example 2. B-ya E.D. 2003 year with a diagnosis: Pathological destructive dislocation of the right femur with deformation of the remaining stump of the head and neck of the femur in the form of a "herding stick". Condition after several attempts at conservative reduction. Figure $2(a, b)$.

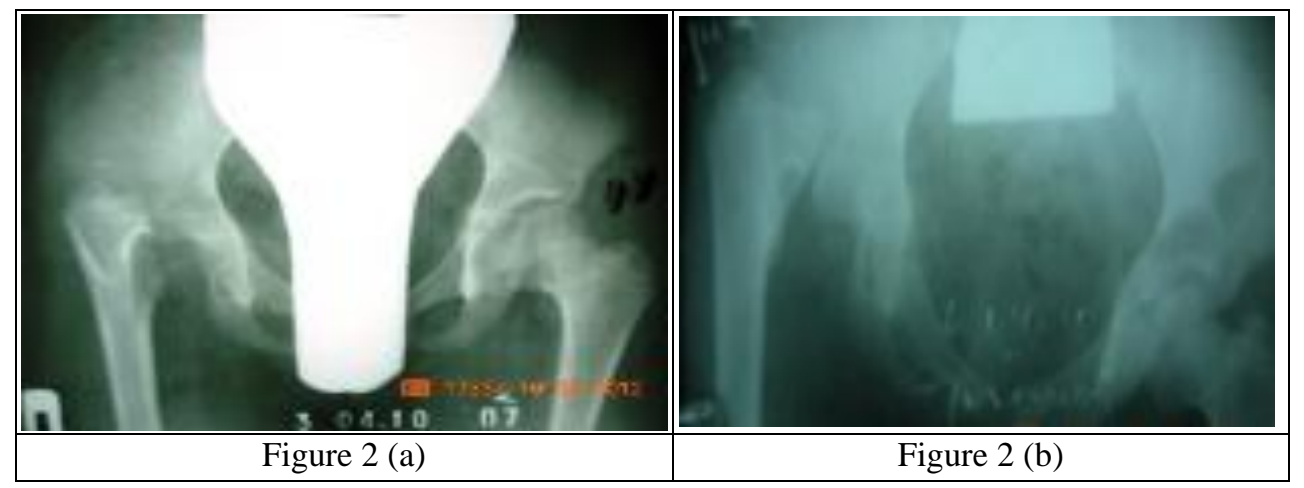

Figure 2 (a). Pathological destructive dislocation of the right femur with deformation of the proximal femur. Figure 2 (b). X-ray of the hip joints in the position of internal rotation of the right lower extremity: deformation of the remaining stump of the head and neck of the femur in the form of a "herding stick".

At the first stage, an open reduction of the stump of the head and neck of the right femur was performed with modulating resection of the last and intertrochanteric shortening detorsion-valgizing and "lengthening" neck osteotomy according to the above-described technique.

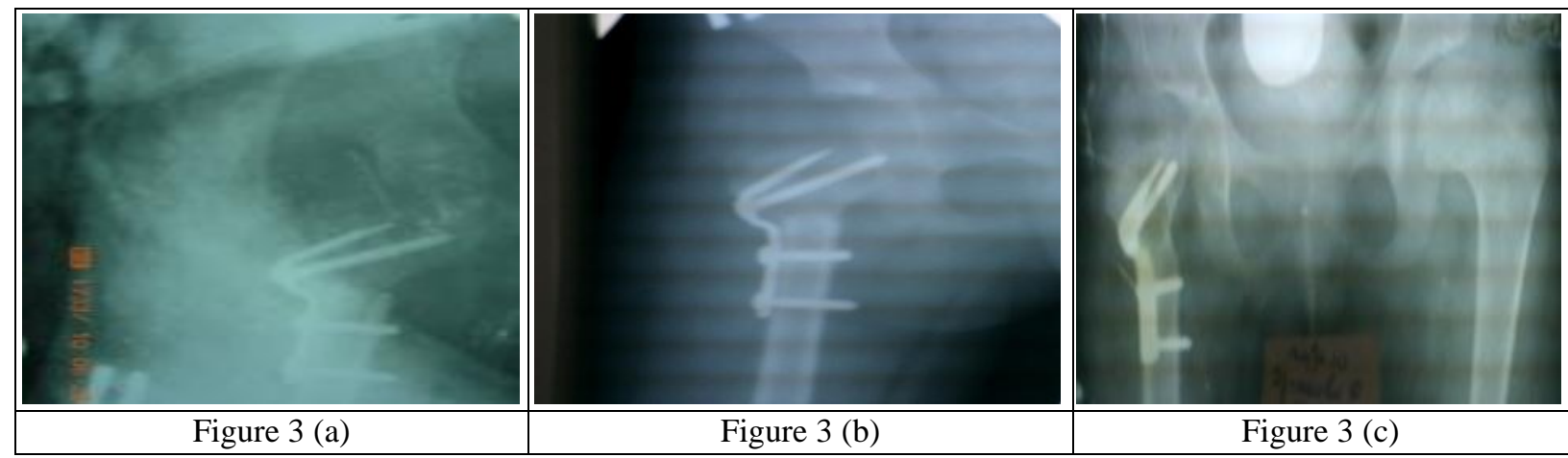

Figure 3 (a). SPO. Open reduction of the stump of the head and neck of the right femur with modulating resection of the last and intertrochanteric shortening detorsion-valgus and "lengthening" neck osteotomy according to the abovedescribed technique.

Figure 3 (b). SPO. 1.5 months after removing the hip plaster cast.

Figure 3 (c). SPO. 8 months before removal of the hardware from the proximal end of the right femur.

At the second stage, under combined anesthesia, the hardware was removed from the proximal end of the right femur. As a result of the treatment, the supportability of the right lower limb was achieved. The child walked with crutches 3 months after the removal of the metal structure, then walked independently without limping on the right 
lower limb. Trendelenburg's symptom was negative. In the right hip joint, the movements reached a satisfactory volume.

\section{Results and Discussion}

All patients underwent surgical treatment. When choosing the tactics of surgical treatment, we proceeded from the age, nature, and severity of the dislocation in both joints. Treatment outcomes were assessed according to the achievement of anatomical and functional outcomes. Dysfunction of the gluteal muscles due to the high position of the greater trochanter, taking into account its pathogenesis and the patient's age, we propose to correct simultaneously with the direction of the position of the cervical deformity in the intertrochanteric region in order to deviate the greater trochanter posteriorly (Verrall et al., 2007; Stagni et al., 2000; Tjiang \& Sidiartha, 2018). A decrease in the distance between the greater trochanter of the femur, the cartilaginous edge of the acetabulum, and the ilium leads to their mechanical conflict, first during abduction and extension of the thigh, and in severe cases, in the middle position. In children under 9 years of age, in order to correct the deformity of the proximal femur, compensate for the lost elements of the femur, restore and stabilize the articular ratio, we recommend performing an intertrochanteric detorsion-shortening osteotomy of the femur with "apophysiodesis" to stop further growth of the greater trochanter, and in children over 9 years of age with progressive Trendelenburg gait and pain syndrome due to severe hypertrophy of the greater trochanter and negative ATD value with "bringing down" the greater trochanter of the femur in the caudal direction. In this case, the greater trochanter will be slightly lower projection all $(2.0-2.5 \mathrm{~cm})$, and the ratio between the anterior and posterior bundles of the gluteus maximus muscle will also change, which determines horizontal instability under the prevailing conditions (Matsuda, 2009; Khanna et al., 2014; Malkani et al., 2019).

\section{Conclusion}

The method of surgical treatment of pathological hip dislocation developed by us, which provides for intertrochanteric detorsion-shortening osteotomy of the femur with "apophysiodesis" or "bringing down" of the greater trochanter of the femur in the caudal direction ensures the correct centering of the remaining stump of the head and neck of the femur in the space cavity, restoration of the correct components of the femoral head and neck of the hip joint with an improvement in the supporting and dynamic function of the latter in combination with the plastic of the acetabulum roof The restoration of the correct spatial relationships of the components of the hip joint, contributing to the dynamic development of the hip joint, is performed after the fusion of bone fragments, which allow, according to indications, to successfully eliminate the observed shortening of the limb due to lengthening of the hip. indicate that reconstructive operations in most patients eliminate flexion-graft external and external rotational contracture, provide stability of the hip joint and thereby improve the patient's gait and statics, reduce pelvic distortion and eliminate the Trendelenburg symptom (Kawaguchi et al., 2001; Tibor \& Sekiya, 2008). The experience of surgical treatment of patients with pathological dislocations of the hip has shown that the most favorable outcomes are achieved with the use of open reduction of the stump of the head or neck of the femur with intertrochanteric detorsion shortening and varizing osteotomy with rotational osteotomy of the pelvis according to Salter and plasty of the acetabulum according to Pemberton.

\section{References}

Abrishami, S., Karami, M., Karimi, A., Soufali, A. P., Aslani, H. R., \& Badizadeh, K. (2010). Greater trochanteric preserving hip arthroplasty in the treatment of infantile septic arthritis: long-term results. Journal of children's orthopaedics, 4(2), 137-141.

Baghdadi, T., Saberi, S., Eraghi, A. S., Arabzadeh, A., \& Mardookhpour, S. (2012). Late sequelae of hip septic arthritis in children. Acta Medica Iranica, 463-467.

Belokrylov, N. M., Gonina, O. V., \& Polyakov, N. V. (2007). Restoration of oporability in case of pathological hip dislocation as a result of osteolysis of his neck and head in childhood. Traumatology and orthopaedics of Russia, 1, 63-67.

Chang, C. H., \& Huang, S. C. (1997). Reconstruction for sequelae of septic hip in children. Journal of the Formosan Medical Association= Taiwan yi zhi, 96(5), 353-358.

Choi, I. H., Shin, Y. W., Chung, C. Y., Cho, T. J., Yoo, W. J., \& Lee, D. Y. (2005). Surgical treatment of the severe sequelae of infantile septic arthritis of the hip. Clinical Orthopaedics and Related Research $₫, 434,102-109$. 
Choi, I. H., Yoo, W. J., Cho, T. J., \& Chung, C. Y. (2006). Operative reconstruction for septic arthritis of the hip. Orthopedic Clinics, 37(2), 173-183.

Dobbs, M. B., Sheridan, J. J., Gordon, J. E., Corley, C. L., Szymanski, D. A., \& Schoenecker, P. L. (2003). Septic arthritis of the hip in infancy: long-term follow-up. Journal of Pediatric Orthopaedics, 23(2), 162-168.

Forlin, E., \& Milani, C. (2008). Sequelae of septic arthritis of the hip in children: a new classification and a review of 41 hips. Journal of Pediatric Orthopaedics, 28(5), 524-528.

Garkavenko, Y. E. (2017). Bilateral pathological hip dislocation in children. Pediatric traumatology, orthopaedics and reconstructive surgery, 5(1), 21-27.

Goel, S. C., \& Logani, V. (2003). Management of sequelae of septic arthritis of the hip in infancy. $J K$ PRACTITIONER, 10(3), 169-175.

Kawaguchi, M., Hashizume, K., Iwata, T., \& Furuya, H. (2001). Percutaneous radiofrequency lesioning of sensory branches of the obturator and femoral nerves for the treatment of hip joint pain. Regional anesthesia and pain medicine, 26(6), 576-581. https://doi.org/10.1053/rapm.2001.26679

Khanna, V., Harris, A., Farrokhyar, F., Choudur, H. N., \& Wong, I. H. (2014). Hip arthroscopy: prevalence of intraarticular pathologic findings after traumatic injury of the hip. Arthroscopy: The Journal of Arthroscopic \& Related Surgery, 30(3), 299-304. https://doi.org/10.1016/j.arthro.2013.11.027

Kruczynski, J. (1996). Avascular necrosis of the proximal femur in developmental dislocation of the hip: incidence, risk factors, sequelae and MR imaging for diagnosis and prognosis. Acta Orthopaedica Scandinavica, 67(sup268), 3-48.

Krys'-Pugach, A. P. (2008). guk ju. N., Kutsenok ya. B., Darovskii AS Our experience in surgical treatment of orthopedic consequences of purulent coxitis in children. Travma. Trauma, 1, 66-70.

Lew, D. P., \& Waldvogel, F. A. (2004). Osteomyelitis. The Lancet, 364(9431), 369-379. https://doi.org/10.1016/S0140-6736(04)16727-5

Malkani, A. L., Himschoot, K. J., Ong, K. L., Lau, E. C., Baykal, D., Dimar, J. R., ... \& Berry, D. J. (2019). Does timing of primary total hip arthroplasty prior to or after lumbar spine fusion have an effect on dislocation and revision rates?. The Journal of arthroplasty, 34(5), 907-911. https://doi.org/10.1016/j.arth.2019.01.009

Matsuda, D. K. (2009). Acute iatrogenic dislocation following hip impingement arthroscopic surgery. Arthroscopy: The Journal of Arthroscopic \& Related Surgery, 25(4), 400-404. https://doi.org/10.1016/j.arthro.2008.12.011

Menschik, F. (1997). The hip joint as a conchoid shape. Journal of biomechanics, 30(9), 971-973. https://doi.org/10.1016/S0021-9290(97)00051-1

Philippon, M. J., Kuppersmith, D. A., Wolff, A. B., \& Briggs, K. K. (2009). Arthroscopic findings following traumatic hip dislocation in 14 professional athletes. Arthroscopy: The Journal of Arthroscopic \& Related Surgery, 25(2), 169-174. https://doi.org/10.1016/j.arthro.2008.09.013

Pidhaietskyi, V. M., Gayko, G. V., Kozak, R. A., \& Nizalov, T. V. (2021). Infectious complications of hip arthroplasty: Causes and results of treatment. International Journal of Health Sciences, 5(3), 313-320. https://doi.org/10.53730/ijhs.v5n3.1520

Rozbruch, S. R., Paley, D., Bhave, A., \& Herzenberg, J. E. (2005). Ilizarov hip reconstruction for the late sequelae of infantile hip infection. JBJS, 87(5), 1007-1018.

Sokolovsky, A. M., \& Sokolovsky, O. A. (1997). Pathological hip dislocation.

Stagni, R., Leardini, A., Cappozzo, A., Benedetti, M. G., \& Cappello, A. (2000). Effects of hip joint centre mislocation on gait analysis results. Journal of biomechanics, 33(11), 1479-1487. https://doi.org/10.1016/S00219290(00)00093-2

Tibor, L. M., \& Sekiya, J. K. (2008). Differential diagnosis of pain around the hip joint. Arthroscopy: The Journal of Arthroscopic \& Related Surgery, 24(12), 1407-1421. https://doi.org/10.1016/j.arthro.2008.06.019

Tjiang, N., \& Sidiartha, I. G. L. (2018). Lipid profile in obese children with and without insulin resistance. International Journal of Health Sciences, 2(2), 9-17. https://doi.org/10.29332/ijhs.v2n2.110

Verrall, G. M., Slavotinek, J. P., Barnes, P. G., Esterman, A., Oakeshott, R. D., \& Spriggins, A. J. (2007). Hip joint range of motion restriction precedes athletic chronic groin injury. Journal of Science and Medicine in Sport, 10(6), 463-466. https://doi.org/10.1016/j.jsams.2006.11.006 